\title{
ACUTE STIMULATION OF VAGUS NERVE MODULATES BRAIN NEUROTROPHINS, AND STIMULATES NEURONAL PLASTICITY IN THE HIPPOCAMPUS OF ADULT MALE RATS
}

\author{
Pamela Rosso $^{1}$, Marco Fiore ${ }^{1}$, Elena Fico ${ }^{1}$, Angela Iannitelli ${ }^{2}$, and Paola Tirassa ${ }^{*}$ \\ ${ }^{1}$ National Research Council (CNR), Institute of Biochemistry and Cell Biology (IBBC), Rome, Italy \\ ${ }^{2}$ Department of Biotechnological and Applied Clinical Sciences, University of L'Aquila, L'Aquila, Italy.
}

The present study was aimed at evaluating whether single intermittent acute cervical vagus nerve stimulation (ACVS), provided at a frequency which exhibits a clinical efficacy, may influence brain neurotrophins and hippocampal plasticity.

With this purpose, the brain of adult male rats undergoing ACVS was used to analyze the expression of Nerve Growth Factor (NGF) and Brain-Derived Neurotrophic Factor (BDNF) in brain areas known to synthetize these growth factors, and the expression the neural cell adhesion molecule (NCAM), the synaptophysin (SYP) and biosynthetic GABA (GAD67) in the hippocampus.

The effects of ACVS on NGF and BDNF protein and $m R N A$ in hippocampus, hypothalamus and cortex two hours after stimulation were shown to be dependent on the frequencies of ACVS stimulation. Prolonged (three days post stimulation) modifications of NGF and BDNF were also observed in the hippocampus of ACVS rats. An early enhancement of the plasticity markers NCAM, SYP and GAD67 was also found in ACVS hippocampus. Three days after stimulation, NCAM and GAD67 levels were still higher than controls. Immunohistochemistry confirms the stimulatory effects of ACVS on GABA showing an increase in GAD67-positive cells in the dentate gyrus and CA3 hippocampal areas. This study shows that ACVS affects brain NGF and BDNF synthesis in a frequency-dependent manner. Neurotrophins changes are associated with increased hippocampal plasticity, as demonstrated by the observed molecular and morphological modifications. These findings support the role of brain neurotrophins in the ACVS mechanism of action. Biomed Rev 2019;30:99-109

Keywords: vagus nerve stimulation, NGF, BDNF, NCAM, GABA, psychiatry

Received 30 October 2019, revised 19 November 2019, accepted 20 November2019.

*Correspondence to: Dr Paola Tirassa, National Research Council (CNR), Institute of Biochemistry and Cell Biology (IBBC), Rome, Italy; Tel.. +39 06 49976742; E-mail: paola.tirassa@cnr.it 


\section{INTRODUCTION}

The electrical stimulation of the cervical portion of the left vagal nerve (CVS) is an approved treatment for epilepsy and depression (1), and it is currently investigated in animals and humans because of its anti-inflammatory and anti-nociceptive action. In addition, the evidence that vagus nerve stimulation (VNS) leads in enhanced memory in both animals $(2,3)$ and humans $(4,5)$, lead to the idea that CVS might be also an effective treatment for neurodegenerative diseases associated with cognitive decline. In spite of a large number of clinical investigations, the research into CVS's mechanism of action is fragmentary and relatively recent.

Electrophysiological studies in animals have indicated that CVS affects brain functions by a direct action on the nucleus of the solitary tract, which in turn sends projections to several areas including the cerebral cortex, the hippocampus, and the hypothalamus (6-7).

Emission tomography and magnetic resonance confirmed that, together with brainstem, forebrain structures, such as the hippocampus are activated in humans (8) and rats (9) by VNS.

Studies reporting the effect on memory retention $(2,4)$ and the enhancement of the long-term potentiation $(10,11)$ after acute stimulation, further indicate the involvement of hippocampus and suggest that VNS may trigger factors, and/ or events which regulate the neural activity and the activitydependent hippocampal plasticity.

The neurotrophins, Nerve Growth Factor (NGF) and Brain Derived Neurotrophic Factor (BDNF) are largely known as potent regulators of brain neuronal activity. Changes of their synthesis or release are demonstrated to occur because of physiological and pathological challenges, including inflammation $(12,13)$. Stress, mood and neurodegeneration are also associated with alterations of neurotrophins levels in the brain (14). The anti-depressant efficacy of anti-neurodegenerative treatments is strictly associated and/or dependent on the rebalance or enhancement of brain neurotrophins-producing areas such as hippocampus, hypothalamus, and cortex $(14,15)$.

To the present day, only a few data are available on the effects of VNS on brain neurotrophins. By using implantable stimulatory devices, which stimulate continuously the vagus nerve, it was found that BDNF and NGF mRNAs in hippocampus and cortex were increased and decreased respectively (17). Biggio and colleagues (18) using the same stimulatory parameters reported different effects of acute (3 hours) or chronic (1 month) VNS on BDNF levels and hippocampal plasticity.

Several human and animal studies demonstrated that the anti-inflammatory, anti-depressant or anti-epileptic effects as well as the activation of subcortical brain areas, including the hippocampus and hypothalamus, and the cortex, produced by vagal nerve, are dependent on stimulation frequency (19). Beside this, the present study addresses the questions whether acute cervical vagus nerve stimulation (ACVS) at a frequency exhibiting clinical efficacy in rats (2, 17-19), might affects neurotrophins expression in the brain, and influence hippocampal synaptic plasticity and memory, such as the neural cell adhesion molecule (NCAM) and the synaptophysin (SYP). In addition, since GABAergic neurons excitation promotes activity-dependent neurotrophins synthesis or release in the hippocampus $(20,21)$, and contributes to the clinical efficacy of CVS as anti-epileptic treatment $(17,23)$, the effects on the distribution and expression of GAD67 were also analyzed.

\section{MATERIALS AND METHODS}

\section{Animals, surgical procedures and electrical stimulation}

Adult male Sprague-Dawley rats (260-320 g b. w.) were used in this study. The rats undergoing ACVS were deprived of food for 14-20 hours before surgery and were anaesthetized with injections of a mixture of ketamine $(70 \mathrm{mg} / \mathrm{kg}$ ) and xylazine $(10 \mathrm{mg} / \mathrm{kg})$ to keep the surgical level of anesthesia during stimulation. Body temperature was maintained at $+37.2 \pm 0.1$ ${ }^{\circ} \mathrm{C}$ (ATB-1100, Nihon Kohden, Japan). During surgery, oxygen level was continuously monitored by using pulse oxymeter (model 9847 V, Nonin Medical, Minneapolis, USA).

To perform vagal stimulation, the left cervical segment of vagus nerve was identified, separated from the surrounding tissues and placed on a pair of platinum wire electrodes immersed in paraffin oil (Kebo Lab.), and the distal end of the nerve was crushed (Fig.1A). This procedure was selected to decrease the potential interferences due to signals originated from muscles or tissues surrounding the stimulation area, or by the discharge of vagal efferents.

The nerve was stimulated using a Wavetek function generator (Wavetek, San Diego, CA, USA) providing square-wave monophasic pulses ( 5 or $20 \mathrm{~Hz} ; 2,5 \mathrm{~ms} ; 2,5 \mathrm{~V}$ ). The nerve was stimulated for 4 periods of $30 \mathrm{~s}$ with a 5 -min interval (ACVS). The control group (Sham) was prepared identically, the nerve put on the electrode but no electrical stimulation provided. The other groups included non-stimulated but fasted rats (Fasted) and naïve rats (CTR).

All animal experiments were carried out in accordance with the U.K. Animals (Scientific Procedures) Act, 1986 and associated guidelines, the European Communities Council 


\section{HIPPOCAMPUS}

\section{A}
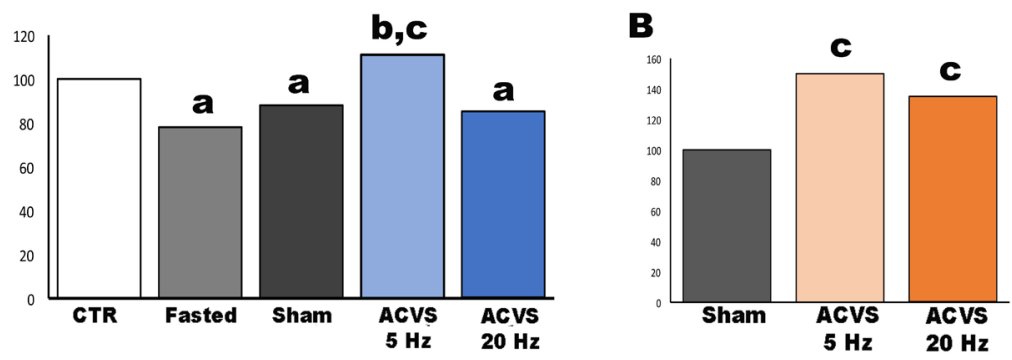

CORTEX
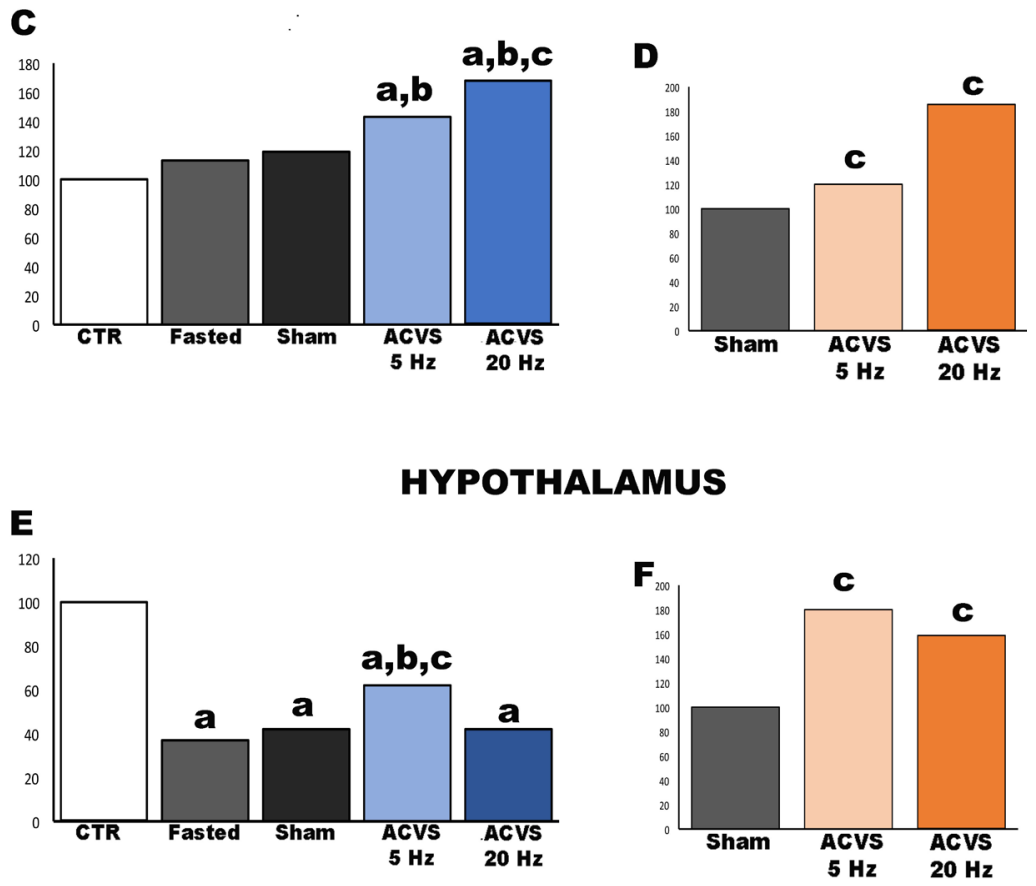

Figure 1. Levels of NGF protein $(A, C, E)$ and $m R N A(B, D, F)$ in the brain areas of hippocampus, cortex and hypothalamus of rats at $120 \mathrm{~min}$ after ACVS. Results are reported as average respect to Control (=100\%). Statistical significance $<0.05 v \mathrm{~s}$ $a=$ Control, $b=$ Fasted and $c=$ Sham.

Directive of 24 November 1986 (86/609/EEC) or the National Institutes of Health guide for the care and use of Laboratory animals (NIH Publications No. 8023, revised 1978), and the intramural ethical committees. All efforts were made to minimize the number of animals used and the occurrence of suffering and injuries.

\section{Experimental design and tissue sample collections}

The experimental design includes a short term and a longterm study, which are referred to as Experiment 1 and Experiment 2 , respectively.
In Experiment 1, four rats from each treatment group were sacrificed (within 120 min from the stimulation) by decapitation. Brains were harvested and hippocampus, hypothalamus and cortex were collected for neurotrophins measurement. Extracts from the hippocampus were further used to analyze the expression of plasticity markers by western blot.

In the Experiment 2, ACVS (20 Hz; 2,5 ms; 2,5 V) and Sham rats were moved to their cages and maintained on a $12 \mathrm{~h}$ light-dark cycle and provided food and water ad libitum for three days before sacrifice. This second experiment explores the late effects of ACVS on hippocampus, by ana- 
lyzing the expression levels of neurotrophins and plasticity markers. The immunohistochemical distribution of GAD67 in hippocampus was also analyzed. To perform the immunohistological studies, Sham and ACVS rats were intracardiacally perfused with $4 \%$ Paraformaldehyde, the brains were removed and post-fixed for $24 \mathrm{~h}$. After one day in sucrose phosphate buffer solution, the brains were cut as described follow.

\section{Tissue analysis}

Proteins were extracted by tissue ultrasonication in Tris-buffer pH 7.00 (Tris-acetate $20 \mathrm{mM} \mathrm{pH}$ 7.5, Nacl 150 mM, EDTA 1 $\mathrm{mM}$, EGTA $1 \mathrm{mM}$, sodium-pyrophosphate $2.5 \mathrm{mM}$, Ortovanadate $1 \mathrm{mM}, \beta$-Glycerolphosphate $1 \mathrm{mM}, \mathrm{NaF} 100 \mathrm{mM}$, PMSF 1 $\mathrm{mM}$, leupeptin $1 \mu \mathrm{g} / \mathrm{ml}$ ) and then centrifuged at $10.000 \mathrm{~g}$. Total protein content was determined in the supernatants by using micro-lowry assay. Protein extracted from the hippocampus, hypothalamus and cortex were used to analyze the effects of ACVS on the expression levels of NGF and BDNF. Extracts from hippocampus were used for western blot analysis.

\section{NGF and BDNF analysis}

Protein extracted form hypothalamus, cortex and hippocampus were used for measuring the NGF and BDNF levels by ELISA assay using the rat b-NGF Duoset Elisa (R\&D Systems, Inc., Minneapolis, MN, USA), following the manufacturer's instructions. The assay is specific for rat b-NGF with approximately $0.1 \%$ and $50 \%$ cross-reactivity with human and mouse recombinant b-NGF respectively, and has no cross-reactivity with other human or rat growth factors. The colorimetric reaction product was measured at $450 \mathrm{~nm}$ using a microplate reader (Dynatech MR 5000; PBI International, Dynatech International, Edgewood, NY, USA). Results are expressed as $\mathrm{pg} / \mathrm{mg}$ protein (mean $\pm \mathrm{SD}$ ).

The total RNA was extracted from the half hippocampal, cortex and hypothalamus tissues by using the TRIzol Kit (Gibco) and complementary DNA (cDNA) was synthesized from $2 \mathrm{mg}$ of total RNA using 200 Units of M-MLV reverse transcriptase (Promega Italia, Milano, Italy) in $20 \mathrm{ml}$ of total volume reaction containing: $250 \mathrm{ng}$ Oligo (dT)12-18 primer, 0.5 Units RNasin ribonuclease inhibitor and $0.5 \mathrm{mM}$ dNTP in $5 \mu 1$ reaction buffer $(250 \mathrm{mM}$ Tris- $\mathrm{Cl} \mathrm{pH} 8.3,375 \mathrm{mM} \mathrm{KCl}$, $15 \mathrm{mM} \mathrm{MgCl} 2,50 \mathrm{mM}$ DTT). The mixture was incubated at $42{ }^{\circ} \mathrm{C}$ for $1 \mathrm{~h}$ and the reaction was terminated with an additional incubation at $95^{\circ} \mathrm{C}$ for $5 \mathrm{~min}$. The cDNAs were used to evaluate the NGF (primers TCCACCCACCCAGTCTTCCA/ GCCTTCCTGCTGAGCACACA) and BDNF (primers
AGCTGAGCGTGTGTGACAGT/TCCATAGTAAGGGCCCGAAC) mRNA expression levels by RT-PCR using GAPDH as housekeeping gene.

\section{Western blot analysis}

Equal protein amounts were loaded on a 10\% SDS-PAGE gel and electrophoretically transferred to nitrocellulose membranes later blocked in low-fat milk $(10 \%$ in Tris buffer and $0.1 \%$ Tween-20) and washed three times for 10 min each at RT in TBS-T. Samples were exposed to the following primary antibodies rabbit polyclonal GAD67, mouse monoclonal antisynaptophysin (SYP), rabbit polyclonal anti-NCAM (1:1000, Merk-Millipore; Italy). The blots were washed and incubated for $1 \mathrm{~h}$ with anti-rabbit or anti-mouse horseradish peroxidase conjugated antibody (Amersham Pharmacia Biotech Italia). These steps were followed by incubation for $1 \mathrm{~h}$ with secondary antibodies. Immunoblot analyses were performed using a chemiluminescence detection kit (ECL) as the chromophore (ECL system by Amersham Bioscience) and exposed to Hyperfilm.

The integrate density (ID) of the bands was recorded and quantified by the public domain image NIH Image processing and analysis program for the Macintosh. The $\beta$-actin band immunoreactivity was used to normalize for sample-to-sample variability.

\section{GAD67 immunohistochemistry}

Sham and ACVS rats were intracardiacally perfused with $4 \%$ Paraformaldehyde, the brains were removed and post-fixed for $24 \mathrm{~h}$. After one day in sucrose phosphate buffer solution, the brains were cut as described follow. Free floating single or double immunostaining was performed on fifteen-micron cryostat sections using monoclonal anti-GAD67 (1:2500, Merk-Millipore; Italy) as primary antibody. The sections were incubated overnight and further processed for immunoperoxidase with the ABC Vectastain Kit (Vector Lab. Inc. Burlingame, USA) following the manufacturer's instructions, using DAB or GS as chromogen. Staining specificity was assessed by omission of the primary antibody and by $\operatorname{IgG}$ isotype. Sections were then washed, mounted on a glass slides, coverslipped and visualized on the Nikon Eclipse E600 equipped with the Nikon DMX 1200 digital camera connected to a PC computer.

\section{Statistical analysis}

Data were obtained by means of analysis of variance (ANOVA). The different controls and CVS conditions were considered as variables. Differences between groups were 
determined by Tukey's post-hoc test: a $\mathrm{p}<0.05$ was considered statistically significant.

\section{RESULTS}

\section{Effect of ACVS on brain NGF and BDNF}

The results of short term effects of ACVS using the low $(5 \mathrm{~Hz})$ and high $(20 \mathrm{~Hz})$ frequencies of stimulation (Experiment 1) show that starvation and ACVS frequencies differentially affect the NGF and BDNF expression levels in the hippocampus, hypothalamus and cortex (Fig. 1 and 2 respectively). More than 16 hours of starvation resulted in decreased NGF and BDNF protein levels in hippocampus and hypothalamus, while no significant effects were noted in the cortex. No differences in brain neurotrophins between Fasted and Sham rats were observed indicating that the surgical procedure per se did not affect NGF and BDNF levels.

As far as the effect of frequency is concerned, we found that following simulation at $5 \mathrm{~Hz}$, increase of NGF protein was observed in all three brain areas examined, while stimulation with $20 \mathrm{~Hz}$ resulted increase and decrease NGF in cortex and hypothalamus (Fig. 1). The NGF mRNA levels were increased in hippocampus, cortex and hypothalamus by ACVS at both frequencies.

The levels of protein and mRNA of BDNF in hypothalamus and cortex was augmented by stimulation with $20 \mathrm{~Hz}$, while no changes were found in the hippocampus (Fig. 2).

Three days after stimulation with $20 \mathrm{~Hz}$, no significant variation of NGF and BDNF proteins in the hippocampus

HIPPOCAMPUS

A

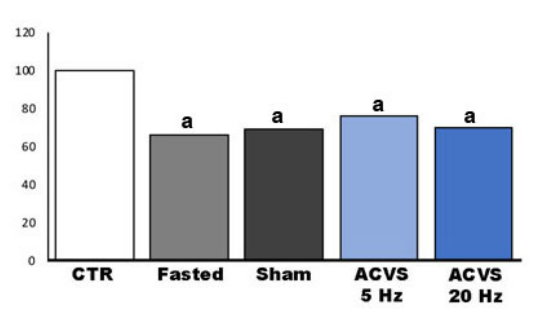

B

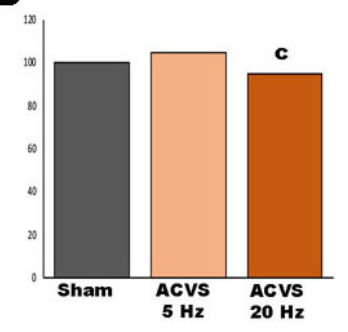

\section{CORTEX}

C

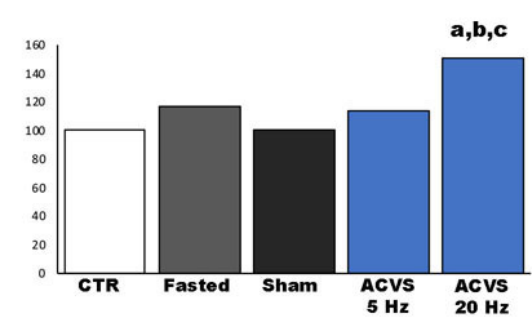

D

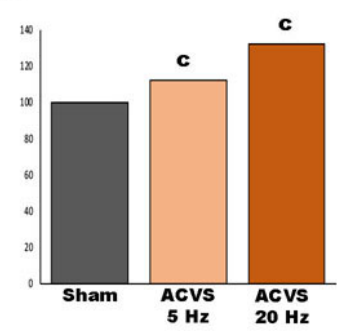

HYPOTHALAMUS

$\mathbf{E}$

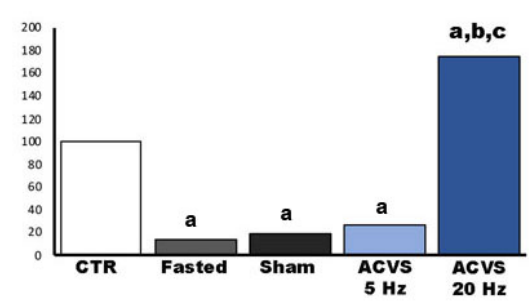

$\mathbf{F}$

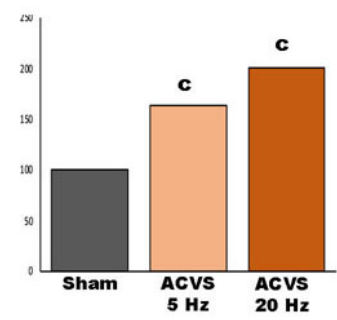

Figure 2. Early effects of ACVS on the levels of $B D N F$ protein $(A, C, E)$ and $m R N A(B, D, F)$ in the brain synthesizing areas. Results are reported as average respect to Control ( $=100 \%)$. Statistical significance $<0.05 v s a=$ Control, $b=$ Fasted, $c=$ Sham. 
of Sham rats were measured. Following ACVS, a significant increase of hippocampal NGF was found, while no significant changes of BDNF were observed (Fig. 3).
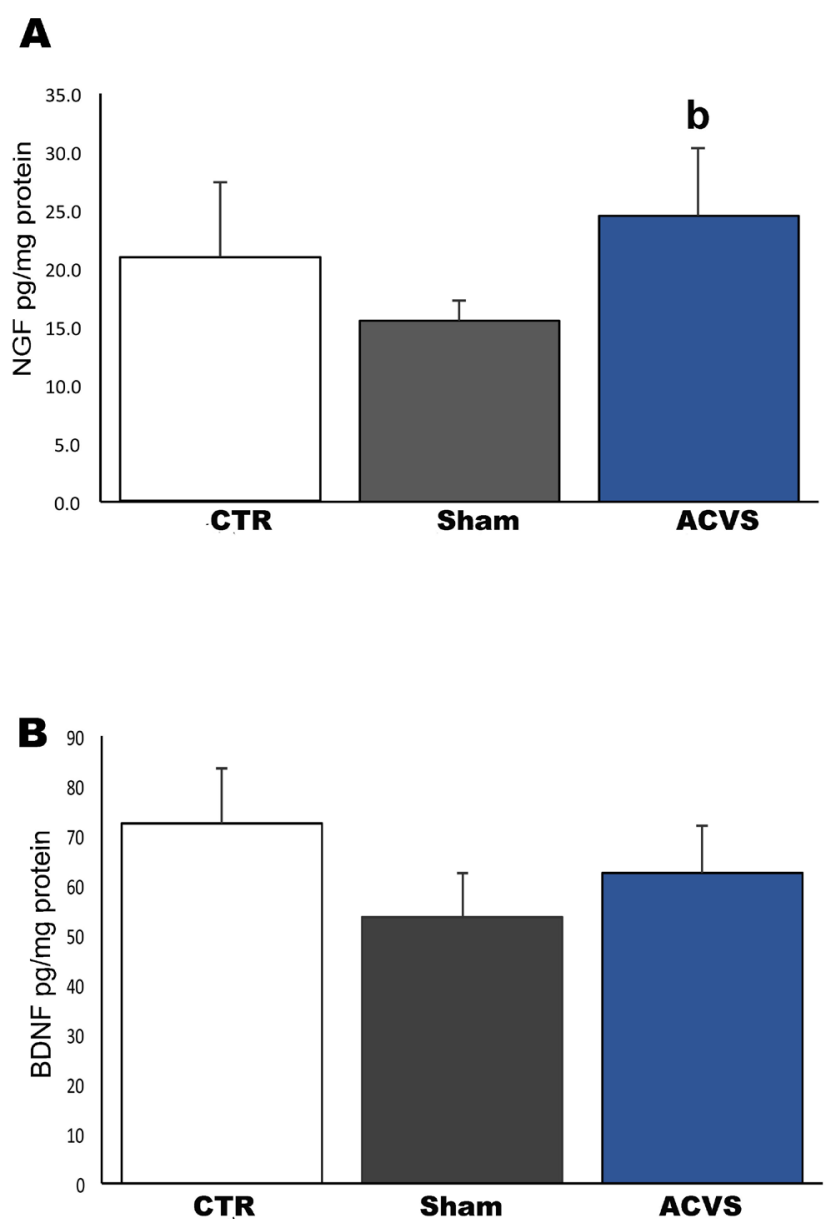

Figure 3. Neurotrophins levels in hippocampus of ACVS rats at three days after stimulation. The values of $N G F(A)$ and $B D N F(B)$ are expressed as $\mathrm{pg} / \mathrm{mg}$ protein, and the data are presented as mean \pm s.d. Statistical significance $<0.05 \mathrm{vs}$ $a=$ Control, $b=$ Sham.

\section{Effects of AVCS on the expression of marker of plasticity in the hippocampus}

The results of the western blot analysis of NCAM, GAD67 and SYP expression in hippocampus of adult rats are shown in Figure 4A-E. Two bands corresponding to the NCAM isoforms of 180 and $140 \mathrm{kDa}$ were found in the hippocampal extracts while no smaller products, including the NCAM isoform at $120 \mathrm{kDa}$, were visible in our experimental condition (Fig. 4A). The band densitometric analysis shows a significant increase of
NCAM $180 \mathrm{kDa}$ but no changes of NCAM $140 \mathrm{kDa}$ in the hippocampus of ACVS when compared to all the control groups (Fig. 4B). Consequently, a significant increase of 180/140 $\mathrm{kDa}$ ratio was also found in ACVS hippocampus with respect to controls. No variations were found comparing the NCAM expression levels of Control, Fasted and Sham rats (Fig. 4C). GAD67 and SYP levels were also enhanced in ACVS rats reaching about four and two times the levels detected in Control and Fasted/Sham rats respectively (Fig. 4D and 4E). Although the mean GAD67 and SYP levels in the hippocampus of Fasted and Sham rats were higher than in controls, this variation did not reach significant levels.

An enhancement of both the 180 and $140 \mathrm{kDa}$ NCAM protein isoforms was also found in the hippocampus of ACVS rats three days after stimulation. Similar levels of the 180 and 140 $\mathrm{kDa}$ NCAM were measured in Fasted rats (ratio $=0,93 \pm 0,1$ ), while a light increase (no significant) was observable in Sham hippocampus (Fig. 5B). An up-regulation of the 180 $\mathrm{kDa}$ NCAM with respect to the $140 \mathrm{kDa}$ NCAM was found in ACVS rats (Fig. 5A and B). No significant changes were observed in the expression levels of SYP in the hippocampus of Sham and ACVS rats with respect to Controls (Fig. 5C).

\section{GAD67 expression and distribution in hippocampus of $A C V S$ rats}

The Experiment 2 demonstrates that the stimulatory effect of ACVS on GAD67 is still evident three days after stimulation. Indeed, the microscopic observation shows an enhancement of GAD67 cell distribution in CA3 hippocampal areas (Fig. 6A and $\mathrm{B}$ ) and in the dentate gyrus (Fig. 6C and D) of rats receiving vagal stimulation compared to Sham. Increased GAD67 immunoreactivity was also found in other brain regions following ACVS, including the cortex and hypothalamus. GAD67positive cells were also localized in the subventricular areas of both lateral and third ventricles (data not showed).

The results of western blot analysis confirm the immunohistochemical data showing an increase of GAD67 in the hippocampus 3d-ACVS, while no significant changes were observed by comparing Sham with control Fasted group (Fig. 6E).

\section{DISCUSSION}

The present study shows that electrical stimulation of the vagus nerve induces neurotrophin expression in brain target regions, and affects hippocampal plasticity.

In line with Follesa et al., (17) we found that vagus nerve 
A

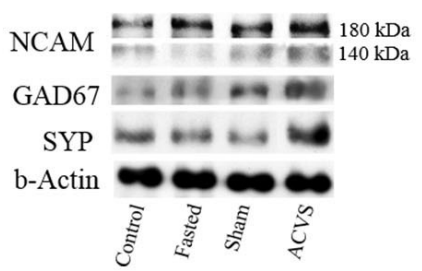

B

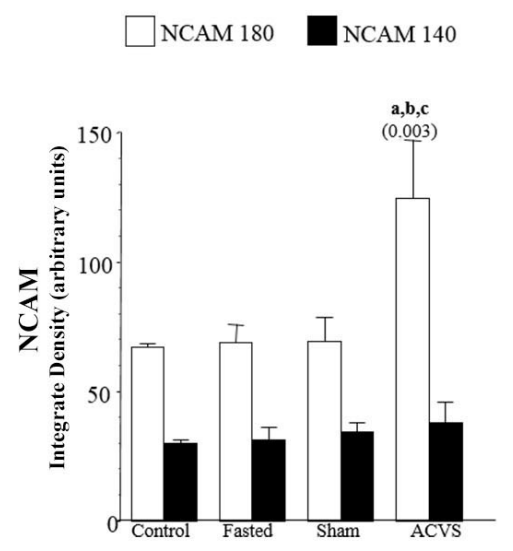

D

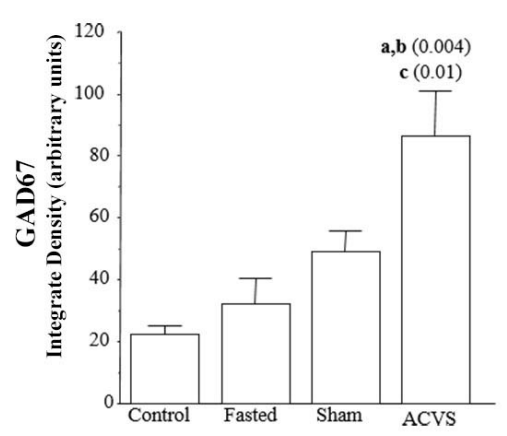

$\mathrm{C}$

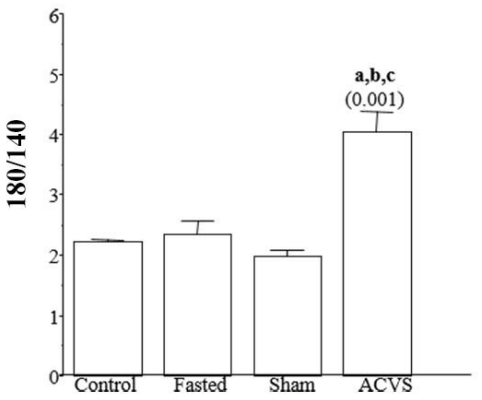

$\mathbf{E}$

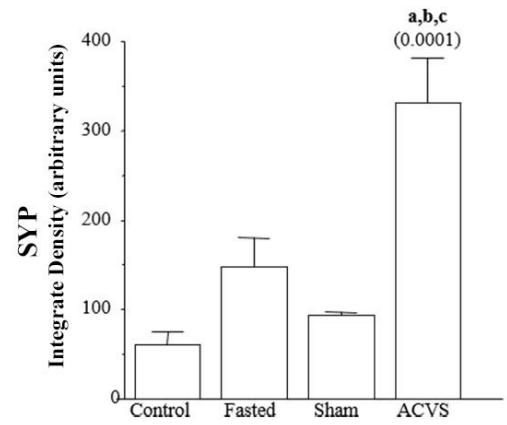

Figure 4. A-E The results of densitometric analyses of NCAM, GAD67 and SYP are reported in graphs B, D, E respectively. Graph $C$ showed the ratio of the 180 and $140 \mathrm{kDa}$ NCAM isoforms. Typical gels showing the effects of ACVS are presented in panel $A$. The values in the graphs are expressed as mean \pm s.d. and the statistical significance is reported in parenthesis: $a=$ Control, $b=$ Fasted and $c=$ Sham.

stimulation modulates brain neurotrophin synthesis. However, at variance with these authors, who stimulated the vagus nerve by a Cybersonic device activated for $3 \mathrm{~h}$ at $30 \mathrm{~Hz}$ in continuous cycle, in our study the electrical stimulation was done on an isolated nerve in anethetized rats avoiding vagal efferent activations and by applying low and medium frequencies and voltage. In this stimulatory condition, we found that ACVS with $5 \mathrm{~Hz}$ causes NGF increase in the hippocampus and hypothalamus, while $20 \mathrm{~Hz}$ were necessary to produce significant changes in brain BDNF.

Humans and animal studies demonstrated that vagus nerve stimulatory parameters and conditions, including frequencies and voltage, duration of treatment, and awaking or sleeping status of the subject might influence the brain response. For example, in depressed and epileptic patients, subcortical regions, including the hypothalamus and hippocampus, respond to low levels of stimulation (pulse width $130 \mu$ s at $1 \mathrm{~Hz}-30 \mathrm{sec}$ on / 180 min off), while high levels of stimulation (pulse width 
A

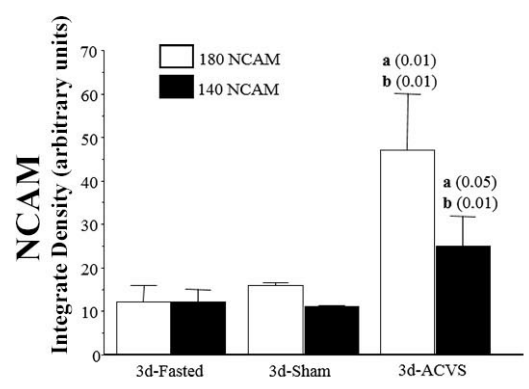

C

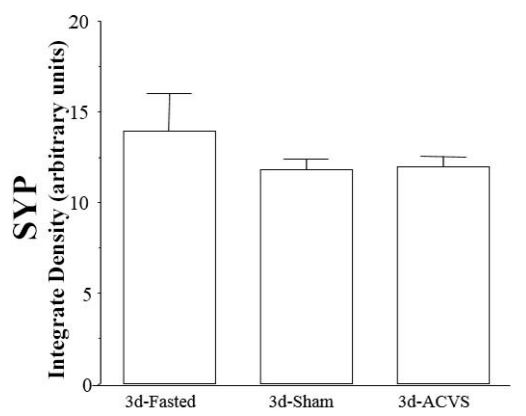

B

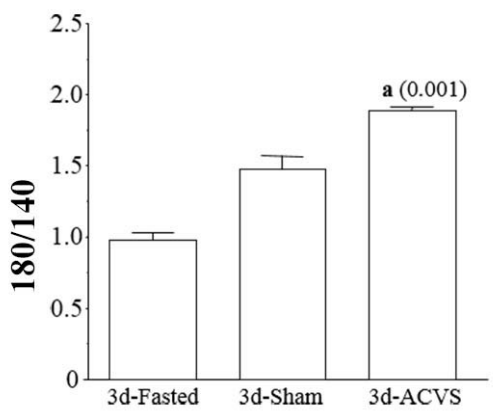

D

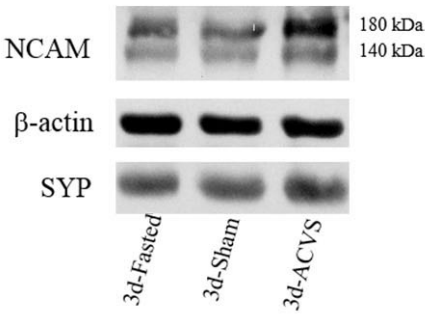

Figure 5. A-D The graphs show the expression level of 180 and $140 \mathrm{kDa} N C A M(A)$, the 180/140 kDa ratio (B) and Synaptophysin (C) in the hippocampus of rats sacrificed three days after surgery and/or stimulation Representative gels are shown in Fig. $3 D$. The values in the graphs are expressed as mean \pm s.d. and the statistical significance is reported in parenthesis: $a=3 d$-Fasted and $b=3 d$-Sham.

Sham
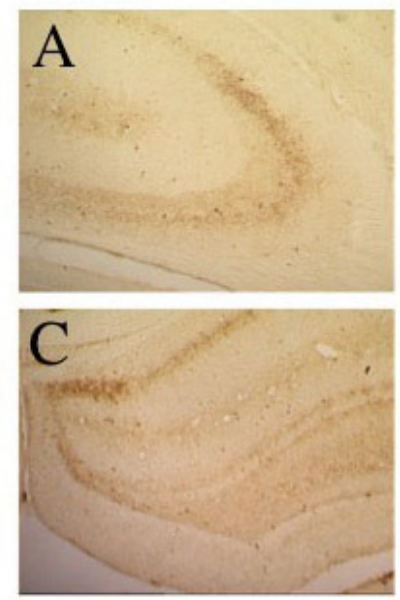

ACVS
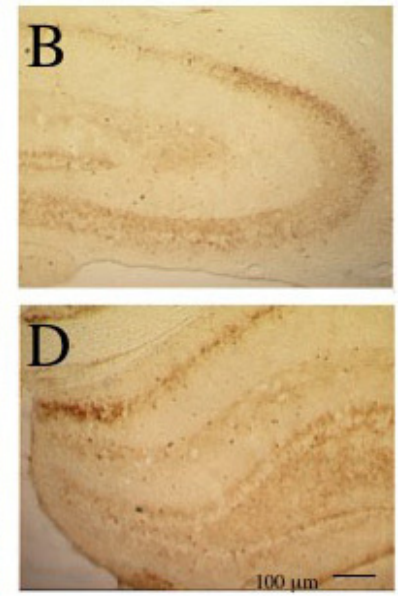

E

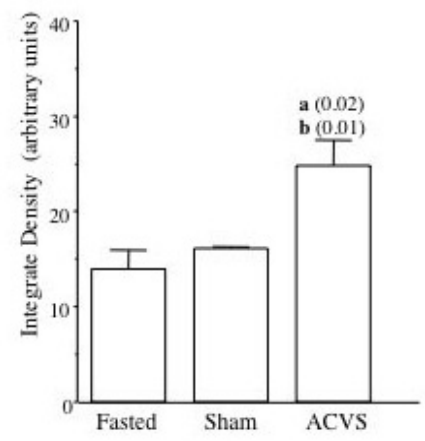

GAD67

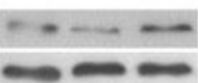

Figure 6. A-E The pictures show the distribution of GAD67 immunoreactivity in the CA3 area ( $A$ and $B$ ) and the dental gyrus $(C$ and $D)$ of the hippocampus of sham (A and $C$ ) and ACVS (B and $D)$. The expression levels of GAD67 (mean \pm s.d.) in hippocampal extracts analyzed by western blot is reported in graph $E$. The statistical difference $v s$ $a=3 d$-Fasted and $b=3 d$-Sham is in parenthesis. 
$500 \mu \mathrm{s}$ at $30 \mathrm{~Hz}-30 \mathrm{sec}$ on / 5 min off) increase the activity in the cortical and prefrontal areas $(25,26)$. A progressive involvement of prefrontal and limbic areas is observable when treatment is prolonged form one month to one year (chronic).

In addition, Krahl et al. reported that the therapeutic efficacy of VNS in awaked rats is mainly due to the activation of A and B- fibers, while higher stimulatory parameters congruent with C-fiber activation are necessary in the anesthetized animals (27), suggesting that the arousal status might be also crucial to develop effective vagus based therapies.

It is therefore likely that the regional specific NGF and BDNF changes obtained by varying the intensity or frequency of stimulation may reflect the recruitment of different vagal fibers and/or sensory pathway activation, and thus the potential anti-inflammatory, anti-depressant or neuroprotective actions of the vagus nerve.

In this context, it is worth to note that the neurotrophin changes by ACVS persist for three days after stimulation and are associated with the induction of plasticity markers and GABA expression in the hippocampus.

Anatomical, electrophysiological and imaging studies have demonstrated that the hippocampus is one of the limbic structures that subserve the neurobehavioral effects of vagus nerve stimulation $(8,9)$. Vagal stimulation enhances long-term potentiation in the dental gyrus of hippocampus (11) and increases synaptic activity in this brain region, as demonstrated by PET analysis (9), further confirming the modulatory role of the vagus nerve on hippocampal activity.

The present study shows that within two hours after ACVS, the hippocampal expression of the molecular plasticity markers $180 \mathrm{kDa}$ NCAM and SYP, and the GABA biosynthetic enzyme GAD67 were enhanced (Experiment 1). Three days after ACVS, the hippocampal levels of GAD67 and NCAM were still increased and these molecular changes were associated with an increased distribution of GAD67-positive nerves in the hippocampus.

The increased expression of SYP $(26,27)$ and $\operatorname{NCAM}(28$, 29 ) in the hippocampus are markers of synaptic plasticity and stabilization following LTP and memory consolidation. The cognitive improvement following physical exercise and environmental enrichment is also characterized by an up-regulation of SYP $(30,31)$ and NCAM (34) and morphological changes in the hippocampus. On the contrary, perturbation of the NCAM expression by genetic or pharmacological methods $(29,33)$ affects behavioral performance and hippocampal plasticity, indicating a direct role of these molecules in the promotion and/or guidance of the morpho-functional rearrangement of the hippocampal network.

Since neurotrophins contribute to synaptic plasticity and their synthesis and release in the hippocampus is also activitydependent (15), it is likely that neurotrophin changes caused by ACVS might reflect the action of the vagus nerve on hippocampal neurons. In line with this suggestion, we observe that ACVS induces an early up-regulation of hippocampal GAD67, which results in a later modification of GABA cell distribution in the hippocampus. Since GABA cells in the brain are neurotrophin targets (36) and synthetize NGF upon stimulation (37), it is likely that ACVS might activate, and/ or potentiate, the brain Neurotrophins/GABA system, which may reflect on normal learning and memory (38) and serve as a self-protective in pathological conditions (39).

Neese et colleague (40) suggested that the vagus nervemediated increase in GAD67-positive cells in the hilum of hippocampus following injury might result by either increased neurogenesis or by an increased commitment of newly born neurons into a GABAergic phenotype. In the hippocampus, the survival of mature and immature neurons as well as the differentiation of neuronal progenitors, including GABA (41), are under control of neurotrophins. It is thus likely that VNSinduced changes on NGF and BDNF synthesis might produce double effects stimulating plasticity and trophism of mature neurons and neurogenesis. This hypothesis is currently under investigation in our laboratory.

In conclusion, our study showing that ACVS modulates neurotrophins synthesis in the brain and results in molecular and structural changes in the hippocampus consolidate the role of the vagus nerve as a mediator of the peripheral-brain network. In addition, our findings that regional selective changes of NGF and BDNF might be induced by varying the stimulatory parameters and conditions offer a new insight into the mechanism of vagal stimulation in neurological and psychiatric diseases.

\section{REFERENCES}

1 Groves DA, Brown VJ. Vagal nerve stimulation: A review of its applications and potential mechanisms that mediate its clinical effects. Neurosci Biobehav Rev 2005;29:493500. doi:10.1016/j.neubiorev.2005.01.004.

2 Clark KB, Krahl SE, Smith DC, Jensen RA. Post-training unilateral vagal stimulation enhances retention performance in the rat. Neurobiol Learn Mem 1995;63:213-216. doi:10.1006/nlme.1995.1024. 
3 Clark KB, Smith DC, Hassert DL, Browning RA, Naritoku DK, Jensen RA. Posttraining electrical stimulation of vagal afferents with concomitant vagal efferent inactivation enhances memory storage processes in the rat. Neurobiol Learn Mem 1998;70:364-373. doi:10.1006/ nlme.1998.3863.

4 Clark KB, Naritoku DK, Smith DC, Browning RA, Jensen RA. Enhanced recognition memory following vagus nerve stimulation in human subjects. Nat Neurosci 1999;2:94-98. doi:10.1038/4600.

5 Sjögren MJC, Hellström PTO, Jonsson MAG, Runnerstam M, Silander HC, Ben-Menachem E. Cognitionenhancing effect of vagus nerve stimulation in patients with Alzheimer's disease: A pilot study. J Clin Psychiatry 2002;63:972-980. doi:10.4088/JCP.v63n1103.

6 Chase MH, Sterman MB, Clemente CD. Cortical and subcortical patterns of response to afferent vagal stimulation. Exp Neurol 1966;16:36-49. doi:10.1016/00144886(66)90084-7.

7 Radna RJ, MacLean PD. Vagal elicitation of respiratorytype and other unit responses in basal limbic structures of squirrel monkeys. Brain Res 1981;213:45-61. doi:10.1016/0006-8993(81)91247-6.

8 Barnes A, Duncan R, Chisholm JA, Lindsay K, Patterson J, Wyper D. Investigation into the mechanisms of vagus nerve stimulation for the treatment of intractable epilepsy, using 99mTc-HMPAO SPET brain images. Eur J Nucl Med Mol Imaging 2003;30:301-305. doi:10.1007/s00259-002-1026-8.

9 Dedeurwaerdere S, Cornelissen B, Van Laere K, Vonck K, Achten E, Slegers G, et al. Small animal positron emission tomography during vagus nerve stimulation in rats: A pilot study. Epilepsy Res 2005;67:133-141. doi:10.1016/j. eplepsyres.2005.09.008.

10 Ghacibeh GA, Shenker JI, Shenal B, Uthman BM, Heilman KM. The influence of vagus nerve stimulation on memory. Cogn Behav Neurol 2006;19:119-122. doi:10.1097/01.wnn.0000213908.34278.7d.

11 Zuo Y, Smith DC, Jensen RA. Vagus nerve stimulation potentiates hippocampal LTP in freely-moving rats. Physiol Behav 2007;90:583-589. doi:10.1016/j.physbeh.2006.11.009.

12 Ciafrè S, Carito V, Ferraguti G, Greco A, Ralli M, Tirassa $\mathrm{P}$, et al. Nerve growth factor in brain diseases. Biomed Rev 2018;29:1-16. doi:10.14748/bmr.v29.5845.

13 Carito V, Ceccanti M, Ferraguti G, Coccurello R, Ciafrè $\mathrm{S}$, Tirassa $\mathrm{P}$, et al. NGF and BDNF Alterations by Prenatal
Alcohol Exposure. Curr Neuropharmacol 2019;17:308317. doi:10.2174/1570159x15666170825101308.

14 Gioiosa L, Iannitelli A, Aloe L. Stress, anxiety schizophrenia and neurotrophic factors: the pioneer studies with nerve growth factor. Riv Psichiatr 2009;44:88-94. doi: $10.1708 / 420.4978$.

15 Castrén E, Antila H. Neuronal plasticity and neurotrophic factors in drug responses. Mol Psychiatry 2017;22:10851095. doi:10.1038/mp.2017.61.

16 Tirassa P, Rosso P, Iannitelli A. Ocular nerve growth factor (NGF) and NGF eye drop application as paradigms to investigate NGF neuroprotective and reparative actions. Methods Mol Biol 2018, 1727:. 19-38. doi:10.1007/9781-4939-7571-6_2.

17 Follesa P, Biggio F, Gorini G, Caria S, Talani G, Dazzi L, et al. Vagus nerve stimulation increases norepinephrine concentration and the gene expression of BDNF and bFGF in the rat brain. Brain Res 2007;1179:28-34. doi:10.1016/J. BRAINRES.2007.08.045

18 Biggio F, Gorini G, Utzeri C, Olla P, Marrosu F, Mocchetti $\mathrm{I}$, et al. Chronic vagus nerve stimulation induces neuronal plasticity in the rat hippocampus. Int $J \mathrm{Neu}$ ropsychopharmacol 2009;12:1209-21. doi:10.1017/ S1461145709000200.

19 Henry TR, Bakay RAE, Pennell PB, Epstein CM, Votaw JR. Brain blood-flow alterations induced by therapeutic vagus nerve stimulation in partial epilepsy: II. Prolonged effects at high and low levels of stimulation. Epilepsia 2004;45:1064-1070. doi:10.1111/j.00139580.2004.03104.x.

20 Woodbury DM, Woodbury JW. Effects of Vagal Stimulation on Experimentally Induced Seizures in Rats. Epilepsia 1990;31:S7-19. doi:10.1111/j.1528-1157.1990.tb05852.x.

21 Roosevelt RW, Smith DC, Clough RW, Jensen RA, Browning RA. Increased extracellular concentrations of norepinephrine in cortex and hippocampus following vagus nerve stimulation in the rat. Brain Res 2006;1119:124132. doi:10.1016/j.brainres.2006.08.048.

22 Tozuka Y, Fukuda S, Namba T, Seki T, Hisatsune T. GABAergic excitation promotes neuronal differentiation in adult hippocampal progenitor cells. Neuron 2005;47:803-815. doi:10.1016/j.neuron.2005.08.023.

23 Ge S, Goh ELK, Sailor KA, Kitabatake Y, Ming GL, Song H. GABA regulates synaptic integration of newly generated neurons in the adult brain. Nature 2006;439:589-593. doi:10.1038/nature04404. 
24 Walker BR, Easton A, Gale K. Regulation of limbic motor seizures by GABA and glutamate transmission in nucleus tractus solitarius. Epilepsia 1999;40:1051-1057. doi:10.1111/j.1528-1157.1999.tb00818.x.

25 Bari AA, Pouratian N. Brain imaging correlates of peripheral nerve stimulation. Surg Neurol Int 2012;3:S260-8. doi:10.4103/2152-7806.103016.

26 Henry TR, Votaw JR, Pennell PB, Epstein CM, Bakay RAE, Faber TL, et al. Acute blood flow changes and efficacy of vagus nerve stimulation in partial epilepsy. Neurology 1999;52:1166-1173. doi:10.1212/wnl.52.6.1166.

27 Krahl SE, Senanayake SS, Handforth A. Destruction of peripheral C-fibers does not alter subsequent vagus nerve stimulation-induced seizure suppression in rats. Epilepsia 2001;42:586-589. doi:10.1046/j.15281157.2001.09700.x.

28 Lynch MA, Voss KL, Rodriguez J, Bliss TVP. Increase in synaptic vesicle proteins accompanies long-term potentiation in the dentate gyrus. Neuroscience 1994;60:1-5. doi:10.1016/0306-4522(94)90197-X.

29 Li S, Reinprecht I, Fahnestock M, Racine RJ. Activitydependent changes in synaptophysin immunoreactivity in hippocampus, piriform cortex, and entorhinal cortex of the rat. Neuroscience 2002;115:1221-1229. doi:10.1016/ S0306-4522(02)00485-2.

30 Senkov O, Sun M, Weinhold B, Gerardy-Schahn R, Schachner M, Dityatev A. Polysialylated neural cell adhesion molecule is involved in induction of long-term potentiation and memory acquisition and consolidation in a fear-conditioning paradigm. $J$ Neurosci 2006;26:10888109898. doi:10.1523/JNEUROSCI.0878-06.2006.

31 Stoenica L, Senkov O, Gerardy-Schahn R, Weinhold B, Schachner M, Dityatev A. In vivo synaptic plasticity in the dentate gyrus of mice deficient in the neural cell adhesion molecule NCAM or its polysialic acid. Eur J Neurosci 2006;23:2255-2264. doi:10.1111/j.14609568.2006.04771.x.

32 Frick KM, Fernandez SM. Enrichment enhances spatial memory and increases synaptophysin levels in aged female mice. Neurobiol Aging 2003;24:615-626. doi:10.1016/S0197-4580(02)00138-0.

33 Marin R, Williams A, Hale S, Burge B, Mense M, Bau- man R, et al. The effect of voluntary exercise exposure on histological and neurobehavioral outcomes after ischemic brain injury in the rat. Physiol Behav 2003;80:167-175. doi:10.1016/j.physbeh.2003.06.001.

34 Kronenberg G, Reuter K, Steiner B, Brandt MD, Jessberger S, Yamaguchi M, et al. Subpopulations of Proliferating Cells of the Adult Hippocampus Respond Differently to Physiologic Neurogenic Stimuli. J Comp Neurol 2003;467:455-463. doi:10.1002/cne.10945.

35 Polo-Parada L, Bose CM, Plattner F, Landmesser LT. Distinct Roles of Different Neural Cell Adhesion Molecule (NCAM) Isoforms in Synaptic Maturation Revealed by Analysis of NCAM $180 \mathrm{kDa}$ Isoform-Deficient Mice. $J$ Neurosci 2004;24:1852-1864. doi:10.1523/JNEUROSCI.4406-03.2004.

36 Porcher C, Medina I, Gaiarsa JL. Mechanism of BDNF modulation in GABAergic synaptic transmission in healthy and disease brains. Front Cell Neurosci 2018;12. doi:10.3389/fncel.2018.00273.

37 Kim J, Lee S, Kang S, Kim SH, Kim JC, Yang M, et al. Brain-derived neurotropic factor and GABAergic transmission in neurodegeneration and neuroregeneration. Neural Regen Res 2017;12:1733-1741. doi:10.4103/16735374.217353.

38 Paulsen O, Moser EI. A model of hippocampal memory encoding and retrieval: GABAergic control of synaptic plasticity. Trends Neurosci 1998;21:273-278. doi:10.1016/ S0166-2236(97)01205-8.

39 Gutiérrez R. The GABAergic phenotype of the "glutamatergic" granule cells of the dentate gyrus. Prog Neurobiol 2003;71:337-358. doi:10.1016/j.pneurobio.2003.11.004.

40 Neese SL, Sherill LK, Tan AA, Roosevelt RW, Browning RA, Smith DC, et al. Vagus nerve stimulation may protect GABAergic neurons following traumatic brain injury in rats: An immunocytochemical study. Brain Res 2007;1128:157-163. doi:10.1016/j.brainres.2006.09.073.

41 Marty S, Berninger B, Carroll P, Thoenen H. GABAergic stimulation regulates the phenotype of hippocampal interneurons through the regulation of brain-derived neurotrophic factor. Neuron 1996;16:565-570. doi:10.1016/ S0896-6273(00)80075-6. 